\title{
CARACTERIZACIÓN AMBIENTAL DE LA CIÉNAGA DE LA QUINTA LOCALIZADA EN CARTAGENA DE INDIAS, COLOMBIA, 2009 - 2010
}

\section{ENVIRONMENTAL CHARACTERIZATION OF THE CIENAGA DE LA OUINTA LOCATED IN CARTAGENA, COLOMBIA, 2009 - 2010}

\author{
Omar Tirado¹, Ganiveth Manjarrez², Claudia Díaz³
}

\begin{abstract}
1 Ingeniero Químico, Esp. Ing Ambiental, Docente Investigador. Fundación Universitaria Tecnológico Comfenalco - Cartagena. Cartagena, Bolívar (Colombia). Barrio España, Carrera 44D No 30ª-91. e-mail: otirado@tecnologicocomfenalco.edu.co 2 Bacterióloga, Microbióloga M.Sc., Coordinadora de Investigaciones, programas ambientales - Fundación Universitaria Tecnológico Comfenalco - Cartagena. Cartagena, Bolívar (Colombia). Barrio España, Carrera 44D No 30ª-91. e-mail: gmanjarrez@tecnologicocomfenalco. edu.co ${ }^{3}$ Ingeniera Civil, Esp. Ing Sanitaria y Ambiental, Docente Investigadora. Fundación Universitaria Tecnológico Comfenalco Cartagena. Cartagena, Bolívar (Colombia). Barrio España Carrera 44D No 30ª-91. e-mail: ing.claudia.diaz@gmail.com
\end{abstract}

Rev. U.D.C.A Act. E Div. Cient. 14(2): 131 - 139, 2011

\section{RESUMEN}

Cartagena de Indias posee diversidad de ecosistemas marinos, los cuales, la convierten en una importante ciudad de Colombia. La Ciénaga de la Quinta es uno de los cuerpos de agua que conforma el sistema hídrico marino lagunar de esta capital caribeña; tiene su propia dinámica hídrica hacia el mercado de Bazurto, con una velocidad de, aproximadamente, $0,06 \mathrm{~m} / \mathrm{s}$, la que es muy baja para dispersar contaminantes. Este cuerpo de agua ha actuado como receptor de disposición final de los residuos del mercado de Bazurto, por lo que, en la actualidad, es evidente su sedimentación y su contaminación por residuos sólidos y líquidos. De igual manera, existe generación de olores ofensivos, vectores y una grave problemática social en torno a éste. La caracterización ambiental del ecosistema, se realizó a través de un estudio exploratorio, utilizando indicadores fisicoquímicos, microbiológicos para las aguas, clasificación de estratos edáficos y cuantificación de metales pesados. Es de resaltar que las concentraciones del oxígeno disuelto, coliformes fecales, materia orgánica y metales pesados, afectan la calidad del agua y sobrepasan los límites admisibles para preservación de flora y fauna en aguas marinas. Así mismo, las condiciones de humedad natural y los contenidos de material orgánica del suelo fueron elevados, generando situación de compresibilidad, evidencias de rellenos y capas de mangle en descomposición.

\section{SUMMARY}

Cartagena de Indias shows a diversity of marine ecosystems, that turns it into an significant town in Colombia. The marsh "la Quinta" is one of the water bodies comprising the ocean water lagoon system of Cartagena, having its own hydric dynamics toward the "Bazurto" market, with a speed of approximately $0.06 \mathrm{~m} / \mathrm{s}$, rate which is very low to disperse pollutants. This water body has acted as a final disposal of waste coming from the Bazurto market, therefore sedimentation and pollution from solid and liquid waste is actually evident. Equally it generates offensive odors, vectors and causes serious social problems in its surroundings. The environmental characterization of this ecosystem was carried out through an exploratory study, using physical-chemical indicators, microbiological ones for water characterization, classification of soil strata and quantification of heavy metals. It is notable to highlight that the concentrations of dissolved oxygen, fecal coliforms, organic matter and heavy metals, affect the water quality and exceeds the permitted limits acceptable for the preservation of flora and fauna in marine waters. Also the conditions of natural moisture and soil organic material content are elevated, generating compressible situation, evidence of land fillings and layers of decaying mangrove.

Key words: Marsh, coliforms, heavy metals.

Palabras clave: Ciénaga, coliformes, metales pesados. 


\section{INTRODUCCIÓN}

Los humedales son ecosistemas dinámicos, cuyo componente fundamental es el agua, en torno, a la cual, se forman ambientes intermedios que varían entre permanentemente inundados y normalmente secos e, incluyen, todos los niveles de diversidad biológica que se puedan sustentar. Sus características físicas se moldean con frecuencia gracias a procesos endógenos, como la sedimentación y la desecación y a procesos exógenos, como inundaciones (Castellanos, 2001).

Los humedales, como las ciénagas, representan numerosos beneficios relacionados con sus funciones ecológicas y ambientales, debido a que sirven de soporte de actividades productivas y socio-culturales y mantienen la biodiversidad (Ministerio de Ambiente, Vivienda y Desarrollo Territorial, 2007).

Colombia cuenta con alrededor de 20 millones de hectáreas de humedales, entre los que se encuentran las ciénagas (Velásquez et al. 2007). En la costa Caribe colombiana, se registra la mayor variación en cuanto a humedales costeros del país se refiere: ribereños, lacustres, palustres, marinos y estuarinos; de amplias playas arenosas y fondos lodosos y forman parte de estos ecosistemas, la ciénaga Grande del Magdalena, la desembocadura de los ríos Sinú y Atrato, la bahía de Cartagena y la Ciénaga de las Quintas, entre muchos otros (Rangel et al. 1997).

El problema ambiental del sistema de caños, de lagos y de ciénagas del Distrito de Cartagena es conocido en el ámbito nacional, desde hace más de medio siglo. Esta problemática ha aumentado, a medida que la capacidad de autorregulación de los cuerpos de agua es insuficiente para degradar la materia orgánica proveniente de las descargas de aguas residuales negras y de residuos sólidos que a estos cuerpos son arrojados (Beltrán E Suárez, 2010).

Uno de los sistemas acuáticos más afectados es la "Ciénaga de la Quinta", que se encuentra en estado deplorable, debido, principalmente, a los impactos provocados por el desarrollo de actividades antrópicas, a pesar de que el informe "Cartagena como Vamos", de 2009, reporta todos sus parámetros en niveles normales.

La ciénaga de la Quinta forma parte integral del sistema de ciénagas y de lagunas de Cartagena y debido a su localización es impactada negativamente por el funcionamiento del mercado de Bazurto. La ciénaga, se comunica con los otros cuerpos de agua de la cual forma parte activa, por medio de las corrientes de intercambio (flujo de materiales) y de la dinámica hídrica (ciénaga de Chambacú, San Lázaro, caño de Juan Angola y Ciénaga de la Virgen), hasta salir al mar, a través de la Bocana estabilizada de mareas. En esta ciénaga, se observan especies de mangle rojo (Rhizophora mangle) y mangle prieto (Avicennia germinans), con funciones de salas cunas de alevinos y otras especies, que hacen de esta ciénaga un ecosistema rico en especies de flora y de fauna. Se resalta que esta flora tiene una función depuradora de las aguas altamente contaminadas de la ciénaga (EPA, Cartagena, 2006); sin embargo, en los últimos años, ha sido objeto de rellenos ilegales e invasiones, loteo y construcción de viviendas. Es de resaltar el caso relacionado con el asentamiento denominado "La islita", ubicada en la Ciénaga de la Quinta y erradicada por la alcaldía de esta ciudad, en el 2008 (Beltrán E Suárez, 2010).

Además, la Ciénaga de la Quinta es utilizada como la principal zona de disposición de residuos de los comercializadores de pescado, de carne y de pollo, quienes indiscriminadamente vierten en esta las vísceras y demás productos, contaminando el agua y generando olores ofensivos. El volumen de residuos producido por el Mercado de Bazurto alcanza cerca de $60 \mathrm{~m}$ o 15 toneladas (Disposición de basuras en Cartagena, 2007).

La recuperación de la zona que rodea el cuerpo de agua, se ha adelantado desde hace cinco años aproximadamente, incluyendo recuperación de espacios públicos ocupados, actividades como dragado, delimitación con cerca, construcción del paseo peatonal y erradicación de residuos sólidos. La orden para la recuperación y el saneamiento específico del cuerpo de agua, fue reiterada en segunda instancia por el tribunal administrativo del Departamento de Bolívar, en noviembre de 2010.

Para la caracterización ambiental del agua, se utilizaron indicadores de calidad ambiental y sanitaria, como los Coliformes totales y fecales (Rosenfeld, 2006; Shibata et al. 2004); además, se cuantificaron niveles de cadmio, plomo y mercurio en muestras de bentos y, para la caracterización del suelo, se realizó clasificación de estratos edáficos, mediante granulometría, límites de Atterberg y contenido de materia orgánica (ASTM, 2008).

\section{MATERIALES Y MÉTODOS}

Ubicación del área de estudio. La Ciénaga de la Quinta hace parte del sistema de caños y lagos internos de la ciudad de Cartagena, como se presenta en la figura 1. Se encuentra localizada entre el puente Jiménez y el puente de Bazurto y se caracteriza por tener anchos entre 288 y $477 \mathrm{~m}$, con profundidad de $2,25 \mathrm{~m}$ y un área total del espejo de agua de 30 hectáreas. 


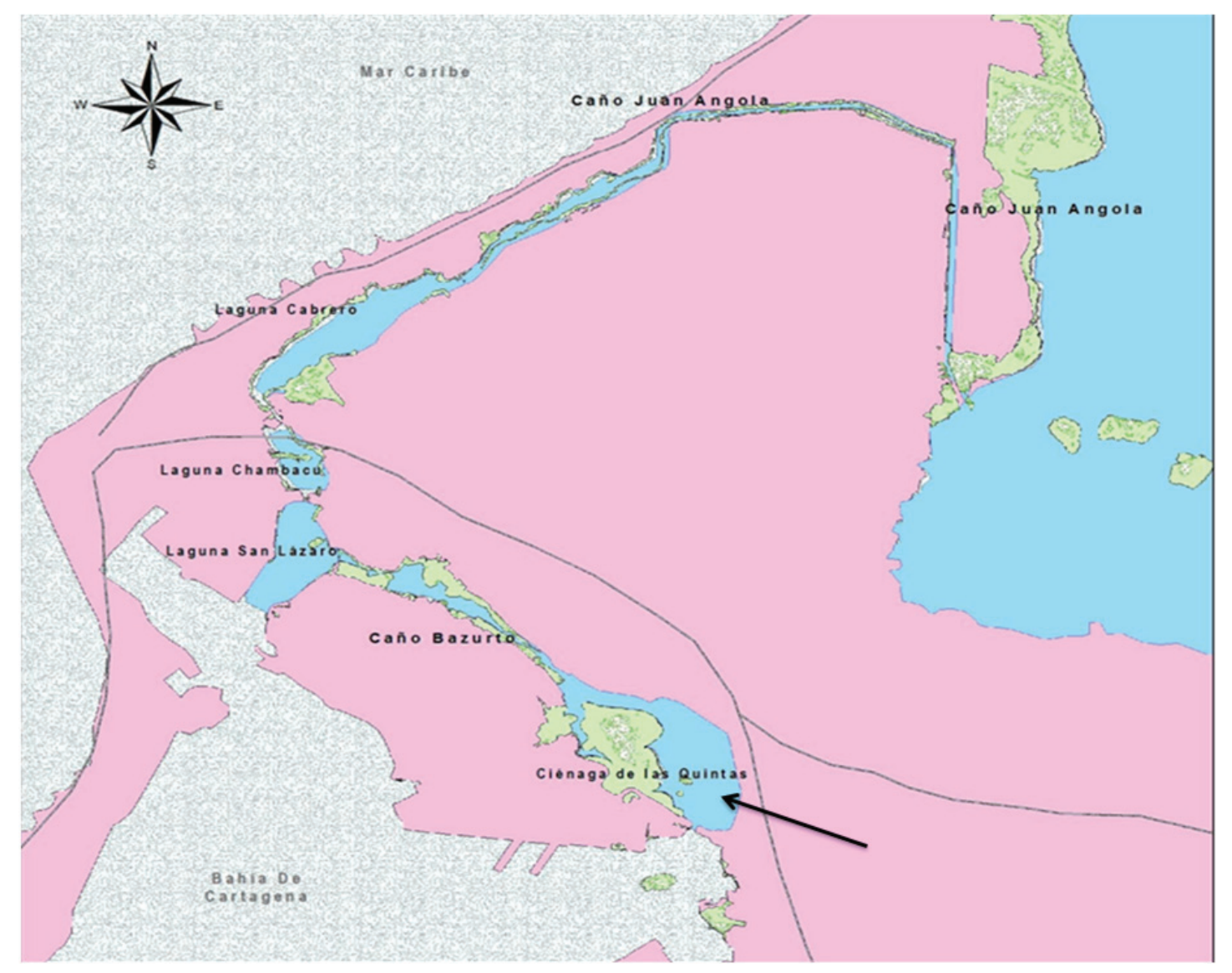

Figura 1. Ubicación geográfica de la zona de estudio (Fuente: Beltrán \& Suárez, 2010).

La ciénaga, se localiza en la latitud $10^{\circ} 24^{\prime} 46^{\prime \prime} \mathrm{N}$ y longitud 75³1'46" E (Ministerio de Ambiente, Vivienda y Desarrollo Territorial, 2007).
Determinación de los puntos de muestreo. Para la caracterización de las aguas de la Ciénaga de la Quinta, se seleccionaron cuatro estaciones estratégicas, cuya georeferenciación se presenta en la tabla 1 .

Tabla 1. Localización de las estaciones de muestreo de agua georeferenciada.

\begin{tabular}{|c|c|}
\hline ESTACIONES & COORDENADAS \\
\hline Estación 1 & $\begin{array}{l}\text { N: } 10^{\circ} 24^{\prime} 46,64 " \\
\text { O: } 75^{\circ} 30^{\prime} 46,79^{\prime \prime}\end{array}$ \\
\hline Estación 2 & $\begin{array}{l}\text { N: } 10^{\circ} 24^{\prime} 41,56 \text { " } \\
\text { O: } 75^{\circ} 31 \text { '31,34” }\end{array}$ \\
\hline Estación 3 & $\begin{array}{l}\mathrm{N}: 10^{\circ} 24^{\prime} 22,22 \text { " } \\
\mathrm{O}: 75^{\circ} 31^{\prime} 24,15 \text { ” }\end{array}$ \\
\hline Estación 4: & $\begin{array}{l}\text { N: } 10^{\circ} 24^{\prime} 20,90^{\prime \prime} \\
\text { O: } 75^{\circ} 31^{\prime} 27,97^{\prime \prime}\end{array}$ \\
\hline
\end{tabular}


Caracterización del recurso agua. Se tomaron muestras de por duplicado en cada uno de los puntos, a una profundidad de $30 \mathrm{~cm}$, aproximadamente, de la superficie. Para ello, se utilizaron botellas de vidrio estériles de $100 \mathrm{~mL}$ de capacidad y transportadas en refrigeración hasta el laboratorio de Ciencias Biológicas de la Facultad de Ingeniería, de la Fundación Universitaria Tecnológico Comfenalco.

Los parámetros físico químicos analizados en cada muestra fueron los siguientes: $\mathrm{pH}$, conductividad, DBO, sólidos disueltos, salinidad y temperatura, utilizando un equipo de medición mutiparámetros: ref, HI 9828 / HANNA.

La determinación de la concentración de Coliformes Totales (CT), se llevó a cabo por medio del recuento indirecto en tubos múltiples de fermentación, expresado en el Número Más Probable (NMP) en 100 mililitros de agua, según recomendaciones de los métodos estándar (APHA, 1998),

Caracterización del recurso suelo. Para el estudio, se realizaron dos perforaciones tipo apiques identificadas como $\mathrm{AP}_{1}$ y $\mathrm{AP}_{2}$ de 0,5 y 1,0 metros de profundidad, en las zonas 2 y 3 (Tabla 1). Cabe resaltar que estos terrenos son el resultado de rellenos sucesivos efectuados en zonas marinas, durante el proceso de expansión de la ciudad (Beltrán E Suárez, 2010).

La caracterización del suelo, se ejecutó por exploración de campo, aplicando métodos manuales, consistentes en perforación, mediante utilización de pala draga y barreno.

Se tomaron muestras cada $0,5 \mathrm{~m}$ hasta llegar a la profundidad máxima de exploración de 1,0m. Las muestras, debidamente almacenadas para conservación de humedad, fueron trasladadas al laboratorio para la ejecución de pruebas de Granulometría (ASTM D421-58 y D422-63), limites de Atterberg (ASTM D423-66, ASTM D 424-59), contenido de humedad y contenido de material orgánico (ASTM D2216-71).

Identificación de metales pesados en bentos. Para el análisis de metales pesados en muestras de bentos, se usaron botellas de Van Dorn, sin fijadores, ni preservativos (McNair $\mathcal{E}$ Chow-Fraser, 2003; Solomon et al. 2011). Además, se utilizó un muestreador de tubo (core samplers), para captar un perfil vertical del sedimento, en aguas someras. Se tomaron cinco muestras aleatorias y se mezclaron en una sola, con el objeto de promediar el valor de metales.

Los metales caracterizados fueron: cadmio, plomo y mercurio. La zona de muestreo, se localizó con un sistema de posicionamiento geográfico (GPS) y fueron equivalentes a las zonas enumeradas, como estación 1, 2, 3 y 4 (Tabla 1).
Posteriormente, estas muestras se llevaron a los laboratorios de la Corporación Autónoma Regional del Canal del Dique - CARDIQUE, para su respetiva caracterización, mediante la metodología del Standar Methods Edición 20 y de digestión ácida (SM3111-B y SM3112 - B).

Análisis de información. Los datos de concentraciones de Coliformes totales, parámetros físico químicos del agua y metales pesados fueron evaluados a través de análisis descriptivo, teniendo en cuenta las condiciones de la zona. Los resultados de metales pesados son expresados en peso seco. La caracterización de suelos, se realizó mediante la descripción de las características físicas encontradas en campo, como textura, estructura y olor; además, se determinó la composición porcentual de las partículas de suelo, con ensayos granulométricos y límites de consistencia, evaluando las condiciones ambientales de recuperación de la muestra.

\section{RESULTADOS Y DISCUSIÓN}

Caracterización fisicoquímica y microbiológica del recurso agua. En las cuatro estaciones de monitoreo, las concentraciones máximas de Coliformes Totales (CT) se presentaron en las estaciones uno y cuatro, con valores de 4,600 y 4,450 NMP/100mL, respectivamente, mientras que los valores más bajos, se encontraron en la estación dos, con una concentración mínima de 1100 NMP/100mL.

En relación con los coliformes fecales (CF), las máximas concentraciones se mostraron en las estaciones uno y cuatro, con valores de 1,500 y $1,900 \mathrm{NMP} / 100 \mathrm{~mL}$, respectivamente.

Es evidente los elevados valores de coliformes totales y fecales, teniendo en cuenta los criterios de calidad de agua admisible para la destinación del recurso para fines recreativos, mediante contacto primario o secundario (Decreto 1594, 1984), ocasionando, posiblemente, problemas de salubridad y de enfermedades de origen hídrico y, más aún, si se tiene en cuenta que en este sector se dan importantes actividades de intercambio comercial y uso de la ciénaga por indigentes.

Las estaciones uno y cuatro se encuentran influenciadas por el aporte de residuos sólidos orgánicos e inorgánicos provenientes de las actividades comerciales del mercado de Bazurto y las malas prácticas higiénicas de la población indigente, que yace alrededor de las mismas.

En la tabla 2, se reúnen los promedios de las determinaciones fisicoquímicas y microbiológicas de las cuatro estaciones de muestreo; en general, los niveles más de $\mathrm{pH}$, temperatura, oxígeno disuelto y salinidad fueron muy heterogéneos en las diferentes estaciones. 
Tabla 2. Resultados promedio de los parámetros fisicoquímicos y microbiológicos en las estaciones de la Ciénaga de las Quinta, 2009 - 2010.

\begin{tabular}{|c|c|c|c|c|c|c|}
\hline Parámetro & $\begin{array}{c}\text { Estación } \\
\mathbf{1}\end{array}$ & $\begin{array}{c}\text { Estación } \\
\mathbf{2}\end{array}$ & $\begin{array}{c}\text { Estación } \\
\mathbf{3}\end{array}$ & $\begin{array}{c}\text { Estación } \\
\mathbf{4}\end{array}$ & Media & DE \\
\hline $\begin{array}{c}\text { Oxígeno } \\
\text { Disuelto (ppm) }\end{array}$ & 4,5 & 7,77 & 4,98 & 4,98 & 5,55 & 1,49 \\
\hline $\mathrm{pH}$ & 7,41 & 4,6 & 7,7 & 8,5 & 7,0525 & 1,69 \\
\hline $\begin{array}{c}\text { Temperatura } \\
\left({ }^{\circ} \mathrm{C}\right)\end{array}$ & 29,18 & 29,09 & 28,91 & 27,8 & 28,745 & 0,63 \\
\hline $\begin{array}{c}\text { Presión atmosférica } \\
\text { (atm) }\end{array}$ & 0,9942 & 0,9942 & 0,9944 & 0,9845 & 0,991 & 0,004 \\
\hline $\begin{array}{c}\text { Conductividad (ms/cm) } \\
\text { Salinidad }\end{array}$ & 39,62 & 43,66 & 41,98 & 43,15 & 42,102 & 1,79 \\
\hline $\begin{array}{c}\text { Sólidos suspendidos } \\
\text { totales (tds Ppt) }\end{array}$ & 19,77 & 19,8 & 20,99 & 18,89 & 19,8625 & 0,862 \\
\hline CT (NMP/ML) & 4,600 & 1,100 & 4,300 & 4,450 & 3,6125 & 1,6794 \\
\hline CF (NMP/ML) & 1500 & 740 & 1120 & 1900 & 1315 & 498,363 \\
\hline
\end{tabular}

Es importante anotar que no existe relación directa entre las variables fisicoquímicas ( $\mathrm{pH}$, temperatura, salinidad y oxígeno disuelto) con la concentración de CT y CF en ninguna de las estaciones, excepto en la estación tres, donde se observó que la salinidad y los sólidos suspendidos mostraron una influencia de tipo regular sobre el grupo de CT y CF $(4,300$ y 1,120 NMP/100mL, respectivamente).

La salinidad presentó una media de $26,25 \%$, considerándose disminuida, si se compara con el estudio realizado por Beltrán E Suárez (2010), desarrollado entre 2007 y 2008, en el cual, determinaron que la salinidad de la Ciénaga oscilaba entre 35 y 36\%, constituyéndose un índice de renovación de aguas; no obstante, el descenso de este parámetro en el 2009 - 2010 podría sugerir poco intercambio hídrico, por parte de la Cienaga.

Los valores de $\mathrm{pH}$ obtenidos en la Cienaga oscilaron entre 4,6 y 8,5 unidades. El valor mínimo, se presentó en la estación dos, correspondiente a la zona justo enfrente del mercado de Bazurto. Teniendo en cuenta que lo estipulado para fines recreativos es un $\mathrm{pH}$ de 5,0 a 9,0 unidades y para la destinación de recurso, con fines de conservación de flora y fauna en aguas estuarinas debe estar entre 6,5 y
8,5 unidades, se señala que esta acidificación podría estar relacionada con los procesos de degradación de residuos sólidos arrojados por los vendedores del mercado, que acidifican las aguas.

Este fenómeno de aguas ligeramente ácidas también fue reportado en la ciénaga de Paticos, en el complejo cenagoso de Ayapel (Córdoba) (Montoya $\varepsilon$ Aguirre, 2009), donde la variable $\mathrm{pH}$ presentó una estratificación definida durante todo el muestreo, con predominio de condiciones cercanas a la neutralidad en la mayor parte de la columna de agua y aguas ligeramente ácidas en la parte inferior de esta, debido a la resuspensión; sin embargo, la mayoría de los estudios reportan ciénagas con aguas alcalinas, como la Cienaga el Eneal, en San Onofre (Sucre) y la Cienaga de Paredes, en Santander (Ríos et al. 2008; Barón et al. 2006).

La concentración de sólidos está representada en la cantidad de materiales suspendidos en las aguas de la Cienaga. Los sólidos suspendidos totales presentaron una media de 19,860 $\pm 0,43 \mathrm{mg} / \mathrm{L}$ y estos resultados pueden estar asociados a la presencia de residuos sólidos, aguas residuales, escorrentías y al suelo arenoso. En el 2007, Beltrán E Suárez (2010) monitorearon este cuerpo de agua, antes de las recientes 
actividades de dragado, teniendo, para entonces, resultados que oscilaban entre 37,548 y 41,062mg/L.

La mayoría de los cuerpos de agua requieren un mínimo de 5 a 6mg/L de oxígeno disuelto (OD). En la Cienaga de la Quinta, los valores oscilaron entre 7,7 y $4,5 \mathrm{mg} / \mathrm{dL}$, permitiendo el soporte de la diversidad de la vida acuática; sin embargo, años atrás se había reportado en esta ciénaga muerte de peces y de aves, como resultado de los valores bajos de OD: 0,2mg/L (Beltrán E Suárez, 2010) lo que trajo consigo presencia de olores ofensivos y disminución de la producción de peces y macroinvertebrados.

Valores bajos de oxígeno disuelto también han sido reportados en horas de la madrugada en la Ciénaga el Eneal, de San Onofre, Sucre (Ríos et al. 2008), en la Ciénaga Colombia, en Caucasia (Velásquez et al. 2007) y en el complejo cenagoso de Ayapel (Montoya E Aguirre, 2009). Teniendo en cuenta la actividad que se ejerce en los alrededores de la Cienaga de la Quinta en horas de la madrugada, sería pertinente el monitoreo del oxígeno disuelto en estos períodos, para obtener puntos de comparación.

La literatura plantea evaluaciones contradictorias de indicadores fecales bacterianos relacionados con diferentes causas ambientales; tal es el caso de Talbert Marsh, un humedal de agua salada de las mareas en el Condado de Orange, junto a dos de las playas más conocidas en el sur de California (Huntington Beach y Newport Beach), donde los niveles de Coliformes están por debajo de los límites permisibles, tanto de CT como de E. coli $(104 \mathrm{y}$ $35 \mathrm{NMP} / 100 \mathrm{~mL}$, respectivamente) (Jeong et al. 2008). En contraste con el presente estudio y al tratarse también de un cuerpo de agua salino, los resultados difieren, probablemente, por el tipo de descargas y por la problemática ambiental y social de los alrededores de la ciénaga de la Quinta.

Un caso a destacar es en Oklahoma, en el llamado Deep Creek, donde el estudio realizado, con el propósito de confirmar los niveles elevados de bacterias coliformes totales y fecales, reveló altos valores: una media de 12,901 \pm 10,817ufc/100mL y $226 \pm 309 u f c / 100 m L$, respectivamente, teniendo en cuenta que la normativa en Oklahoma que regula el uso recreativo del agua es máximo de 126 ufc/100mL, de $E$. coli y de 406 ufc/100mL, para CT (O'Neal E Hollrah, 2007). Es evidente la alta carga bacteriana evidenciada en Oklahoma, debido a que los principales problemas que rodean Deep Creek son la erosión del suelo y la escorrentía (Kistemann et al. 2002). Otros problemas ambientales, como las precipitaciones y los usos del suelo para actividades, como ganadería y agricultura, suelen estar relacionadas con los altos niveles de coliformes, como en el caso de la bahía de Tillamook, una cuenca hidrográfica en Oregon, Estados Unidos (Sullivan et al. 2005).
En Colombia, diversas ciénagas han sido caracterizadas en los últimos años, como la ciénaga Mata de Palma, en el departamento del Cesar (Navia E Estupiñán, 2009); Ciénaga Grande de Santa Marta, en el Magdalena (Narváez et al. 2008); Ciénaga de Ayapel en Córdoba (Chalarca et al. 2007) y Ciénaga Colombia, en Caucasia Antioquia (Velásquez et al. 2007), entre otras. Lo interesante de todas estas caracterizaciones es que, al igual que en nuestro estudio, los valores de CT y CF superan los criterios de calidad de agua admisible para la destinación del recurso para fines recreativos, constituyéndose en importantes focos para la generación de problemas de salubridad y de enfermedades de origen hídrico y más aún si se tiene en cuenta que estos cuerpos de agua están relacionadas con actividades de intercambio comercial.

De acuerdo con los resultados obtenidos en el estudio microbiológico, se recomienda implementar monitoreos continuos de la calidad sanitaria de las aguas de la ciénaga y disminuir la carga de nutrientes que llegan a este cuerpo de agua, procedente de las diferentes actividades económicas desarrolladas en la zona, con el fin de controlar la multiplicación de los microorganismos patógenos y disminuir el riesgo de enfermedades en la población humana y animal aledaña.

Caracterización del recurso suelo: La zona de estudio presenta un tipo suelo homogéneo en cuanto a conformación y características de clasificación, siendo predominante la presencia de limos arenosos grises; en ambos casos, se encontró un fuerte olor a materia orgánica en descomposición, siendo considerablemente mayor en el apique dos. Caso semejante al encontrado en la Ciénaga Colombia, en Caucasia Antioquía, donde se reporta la formación de suelos, conformada por arcillas, por areniscas y por conglomerados estratificados, con suelos bien drenados y profundos (Velásquez et al. 2007).

Los contenidos de humedad encontrados son altos para este tipo de suelos, siendo un hallazgo importante la presencia de mangle en descomposición en mayor proporción en el apique dos, con un contenido de material orgánico superior al $90 \%$, lo que indica la presencia de estratos blandos altamente comprensibles y que sugiere que en esta zona es un área natural de ecosistema de manglar; en oposición a lo anterior, los suelos de la ciénaga de Mallorquín, en el Atlántico, presentan una rápida oxidación de la materia orgánica y poca presencia de material orgánico en los estratos superficiales del suelo (Plan de Ordenación de la Cuenca de la Ciénaga de Mallorquín, 2006).

El nivel freático, se halló en el apique dos, a 0,4m de profundidad. El anterior parámetro es propio de la naturaleza 


\begin{tabular}{|c|c|c|c|c|c|c|}
\hline$\underset{⿱ 乛 龰}{\mathbb{Z}}$ & \multicolumn{2}{|c|}{ 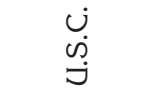 } & $\vec{\varepsilon}$ & $\vec{\varepsilon}$ & $\vec{\varepsilon}$ & $\vec{\varepsilon}$ \\
\hline 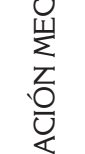 & \multicolumn{2}{|c|}{ 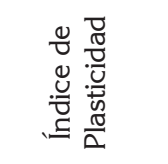 } & 0 & in & $\frac{\hat{z}}{\mathrm{z}}$ & 完 \\
\hline 亗 & \multicolumn{2}{|c|}{ 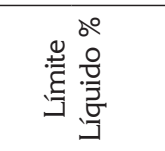 } & 犬্ं & 哭 & $\overrightarrow{\mathrm{z}}$ & $\overrightarrow{\mathrm{z}}$ \\
\hline 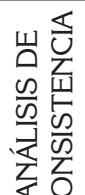 & \multicolumn{2}{|c|}{ 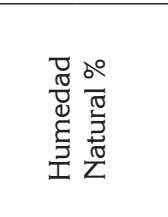 } & $\begin{array}{l}\stackrel{\circ}{\circ} \\
\text { ச } \\
\text {. }\end{array}$ & $\begin{array}{l}\circ 0 \\
8 \\
\text { oे }\end{array}$ & $\begin{array}{l}\text { ه̊ } \\
\text { గి } \\
\text { ని }\end{array}$ & $\begin{array}{l}\text { ठ̊ } \\
\infty \\
\infty \\
\infty\end{array}$ \\
\hline \multirow{8}{*}{ 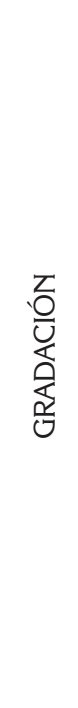 } & \multirow{8}{*}{ 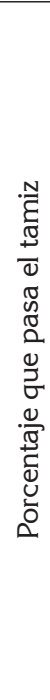 } & ㅇํㅇ & $\begin{array}{l}\circ \\
\infty^{\circ}\end{array}$ & $\begin{array}{l}0 \\
\text { f }\end{array}$ & $\begin{array}{l}0 \\
8 \\
\infty\end{array}$ & $\begin{array}{l}\infty \\
\tilde{N}^{\circ}\end{array}$ \\
\hline & & 음 & $\stackrel{\circ}{\stackrel{0}{N}}$ & $\begin{array}{l}\infty \\
\mathfrak{N}\end{array}$ & $\begin{array}{l}0 \\
\text { î } \\
\infty\end{array}$ & $\begin{array}{l}0 \\
\varnothing \\
\infty\end{array}$ \\
\hline & & $\dot{\circ} \dot{q}$ & $\begin{array}{l}\infty \\
\tilde{\sigma}\end{array}$ & $\hat{\sigma}$ & $\begin{array}{l}0 \\
\vdots \\
\infty\end{array}$ & $\begin{array}{l}\text { 8े } \\
\text { 8े }\end{array}$ \\
\hline & & 운으 & $\vec{a}$ & $\begin{array}{l}+ \\
\text { मे }\end{array}$ & $\begin{array}{l}0 \\
\text { s. } \\
\text { o }\end{array}$ & $\begin{array}{l}0 \\
i \\
\tilde{\sigma}\end{array}$ \\
\hline & & $\dot{\tau} \nabla$ & $\stackrel{8}{-}$ & $\begin{array}{l}\text { oे } \\
\text { ô }\end{array}$ & $\begin{array}{l}\infty \\
\infty \\
\infty \\
\infty\end{array}$ & ถู \\
\hline & & $\frac{\omega}{\dot{m}}$ & & $\stackrel{8}{ }$ & $\begin{array}{l}\forall \\
8 \\
8\end{array}$ & 음 \\
\hline & & ${ }^{N}$ & & & 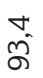 & \\
\hline & & id & & & 8 & \\
\hline \multicolumn{2}{|c|}{ 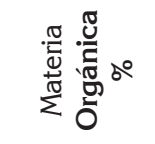 } & & $\begin{array}{l}\text { Oे } \\
\text { ji }\end{array}$ & $\begin{array}{l}n \\
0 \\
0\end{array}$ & $\frac{n}{\sigma}$ & $\begin{array}{l}\text { Oे } \\
\text { बें }\end{array}$ \\
\hline $\begin{array}{l}\frac{5}{w} \\
\frac{w}{\underline{x}}\end{array}$ & $\begin{array}{l}\text { t. } \\
\dot{U} \\
\underline{E}\end{array}$ & $\begin{array}{l}\text { है } \\
\text { Dू } \\
\text { Dે }\end{array}$ & & & & \\
\hline 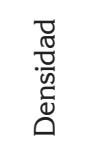 & $\begin{array}{l}\frac{\pi}{0} \\
\text { है } \\
\text { 至 }\end{array}$ & $\begin{array}{l}\text { है } \\
\text { है }\end{array}$ & & & & \\
\hline \multirow{3}{*}{ 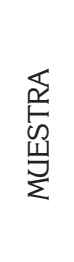 } & \multirow{3}{*}{ 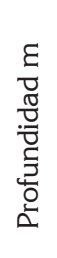 } & $\ll$ & $\stackrel{\text { Ln }}{0}$ & $\stackrel{\circ}{-}$ & $\stackrel{\text { no }}{0}$ & $\stackrel{0}{=}$ \\
\hline & & 㟔 & O. & เి & : & $\stackrel{n}{0}$ \\
\hline & & $\dot{0}$ & - & $N$ & - & $N$ \\
\hline 安 & & $\dot{0}$ & - & - & $N$ & $N$ \\
\hline
\end{tabular}


del suelo, de su capacidad hidráulica y la relación con la existencia de cuerpos de agua cercano, por tal razón es un parámetro importante para el caso puntual de esta ciénaga, pero no amerita comparación con otras ciénagas similares.

Los datos de la caracterización de suelos de la zona son descritos en la tabla 3 .

Caracterización de metales pesados en bentos: La contaminación del ambiente por metales pesados es un serio problema reconocido en el ámbito mundial. Los metales pesados, se destacan por su capacidad de concentración y de bioacumulación, razón que les faculta para ingresar en la cadena trófica (Motas et al. 2003).

Los valores reportados de cadmio $(0,270 \mathrm{mg} / \mathrm{kg})$ están por encima de lo establecido en el decreto 1594 del 84, que regula que para el uso pecuario y destinación del recurso para preservación de flora y fauna, de aguas estuarinas, el criterio de calidad admisible es de $0,01 \mathrm{mg} / \mathrm{kg}$.

En relación con el plomo, los valores encontrados $(27,30 \mathrm{mg} /$ $\mathrm{kg}$ ) son altos al compararlos con el criterio de calidad admisible para aguas de uso pecuario, según lo establecido en el decreto 1594 del 84 , que es de $0,1 \mathrm{mg} / \mathrm{kg}$. Se podría inferir que el origen de este metal en el sedimento se debe a la fabricación de redes de pesca de los moradores del Barrio Chino (Olivero, 2005). En cuanto al mercurio, la cantidad reportada $(0,741 \mathrm{mg} / \mathrm{kg})$ es representativa como metal traza y esta concentración no representa peligro alguno. Las concentraciones del oxígeno disuelto, coliformes fecales y materia orgánica, están afectando, de manera directa, la calidad del agua, admisible para preservación de flora y fauna en aguas marinas.

Lo anterior, permite concluir que la calidad ambiental de las aguas de la Ciénaga de la Quinta, en los actuales momentos, no es apta para contacto primario y no es garantía de supervivencia para la flora y fauna aun existente en las áreas materia de estudio.

Todo tipo de vida depende de la calidad del agua para su supervivencia. Por ende, la protección de este recurso natural debe ser una política local y nacional. Para lograr lo anterior y, al mismo tiempo, un manejo adecuado de este cuerpo de agua, se hace necesario contar con indicadores que permitan evaluar su calidad.

El desarrollo de tales indicadores, se debe hacer con base en las funciones del agua que se evalúa, considerando aquellas propiedades bentónicas sensibles a los cambios del uso del recurso agua en esta zona de la ciudad. En materia de calidad de agua, se requiere ampliar la perspectiva original, enfocada sólo a aguas marinas de carácter lagunar, como lo es la ciénaga de la Quinta, en la ciudad de Cartagena, para incluir también aguas de ecosistemas naturales y modificados, con fines específicos como el urbano.

Conflictos de intereses: El manuscrito fue preparado y revisado con la participación de todos los autores, quienes declaramos que no existe ningún conflicto de intereses que ponga en riesgo la validez de los resultados presentados.

\section{BIBLIOGRAFÍA}

1. APHA. 1998. American Public Health Association, American Water Works Association, Water Pollution Control Federation, 20th Edition, Standard Methods for the Examination of Water and Wastewater. p.1-46.

2. ASTM. 2008. Capacitación en normas. Capacitación en normas para la construcción en México. Disponible desde internet en http://www.astm.org/SNEWS/ SPANISH/SPJF08/outreach_spjf08.html (con acceso 08/10/10).

3. BARÓN, M.; GAVILÁN, R.; RAMÍREZ, J. 2006, Variabilidad espacial y temporal en la comunidad de cladóceros de la Ciénaga de Paredes (Santander, Colombia) a lo largo de un ciclo anual. Limnetica. 25(3):623-636.

4. BELTRÁN, A.; SUÁREZ, L. 2010, Diagnóstico ambiental de los cuerpos internos de agua de la ciudad de Cartagena de Indias, Ed. Eumed. 172p. Disponible desde internet en: www.eumed.net/libros/2.10b/691/ (con acceso el 10/11/10).

5. CASTELLANOS, C. 2001. Los ecosistemas de humedales en Colombia. Revista Luna Azul. 13(7):45-56.

6. CHALARCA, D.; MEJÍA, R.; AGUIRRE, N. 2007. Aproximación a la determinación del impacto de los vertimientos de las aguas residuales domésticas del municipio de Ayapel, sobre la calidad del agua de la ciénaga. Rev. Facultad de Ingeniería. U. de Antioquia. 40:41-58.

7. DECRETO 1594 DEL 26 DE JUNIO DE 1984. Disponible desde internet en http:/www.encolombia. com/medioambiente/hume decreto159484.htm (con acceso el 10/11/10).

8. DISPOSICIÓN DE BASURAS EN CARTAGENA. 2007. Disponible desde internet en http://www.cci.org.co/ publicaciones/1_Oct-05-07\%20Basuras\%20en\%20 Cartagena.pdf (con acceso el 08/10/10). 
9. ENTIDAD PÚBLICA AMBIENTAL. 2006. Disponible desde internet en http://www.epacartagena.gov.co/ doc/caracterizacion-manglares-urbanos-min.pdf (con acceso el 10/10/10).

10. INFORMECARTAGENACÓMOVAMOS.2009. Disponible desde internet en: http://www.cartagenacomovamos. org/temp_downloads/Resumen\%20Ejecutivo\%20 EPC\%202009\%5B1\%5D.pdf (con acceso el 10/09/10).

11. JEONG, Y.; SANDERS, B.; MCLAUGHLIN, K.; GRANT, S. 2008. Treatment of dry weather urban runoff in tidal saltwater marshes: A longitudinal study of the Talbert Marsh in Southern California. Environ. Sci. Technol. 42:3609-3614.

12. KISTEMANN, T.; CLASSEN, T.; KOCH, C.; DANGENDORF, F.; FISCHEDER, R.; GEBEL, J.; VACATA, V.; EXNER, M. 2002. Microbial load of drinking water reservoir tributaries during extreme rainfall and runoff. Appl. Environ. Microbiol. 68(5):2188-2197.

13. MINISTERIO DE AMBIENTE, VIVIENDA Y DESARROLLO TERRITORIAL. 2007. Resolución no. 978. Disponible desde internet en: http://www.minambiente.gov.co/ documentos/res_0978_040607.pdf (con acceso el 10/11/10).

14. MCNAIR, S.; CHOW-FRASER, P. 2003. Change in biomass of benthic and planktonic algae along a disturbance gradient for 24 Great Lakes coastal wetlands. Can. J. Fish. Aquat. Sci. 60: 676-689.

15. MOTAS-GUZMÁN, M.; ROMERO, D.; GARCÍA-DÍAZ, J.L.; GARCÍA-FERNÁNDEZ, A.J. 2003. Niveles de cadmio y plomo en hígado y riñón de équidos para consumo humano. Rev. Toxicol. 20(2):s.p.

16. MONTOYA, Y.; AGUIRRE, M. 2009. Cambios nictemerales de variables físicas y químicas en la Ciénaga de Paticos, complejo cenagoso de Ayapel, Colombia. Rev. Biol. Trop. 57(3):35-646.

17. NARVÁEZ, S.; GÓMEZ, M.; ACOSTA, J. 2008, Coliformes termotolerantes en aguas de las poblaciones costeras y palafíticas de la Ciénaga Grande de Santa Marta, Colombia. Acta Biol. Col. 13(3):115-124.

18. NAVIA, A.; ESTUPIÑ̃́N, S. 2009. Calidad sanitaria del agua de la ciénaga Mata de Palma en el Departamento del Cesar, Colombia. NOVA. 7(11):85-91.
19. OLIVERO, J. 2005, Niveles de plomo en sangre en niños de Cartagena Colombia, 2004 - 2005 Disponible desde internet en http://reactivos.com/metales.html (con acceso el 19/10/10).

20. O'NEAL, S.; HOLLRAH, D. 2007. Monitoring of Total Coliform and Escherichia coli Levels in a Second Order Stream in West-Central Oklahoma. Proc. Okla. Acad. Sci. 87:69-75.

21. PLAN DE ORDENACIÓN DE LA CUENCA DE LA CIÉNAGA DE MALLORQUIÍN. 2006. Disponible desde internet en http://www.crautonoma.gov.co (con acceso el 21/02/011).

22. RANGEL, J.O.; LOWY, P.D.; AGUILAR, M. 1997. Colombia Diversidad Biótica II, Bogotá: Universidad Nacional de Colombia. p.383-402.

23. RÍOS, E.; PALACIO, J.; AGUIRRE, N. 2008. Variabilidad fisicoquímica del agua en la ciénaga El Eneal, reserva natural Sanguaré municipio de San Onofre-Sucre, Colombia. Rev. Fac. Ing. Univ. Antioquia. 46:39-45.

24. ROSENFELD, L. 2006. Temporal and spatial variability of fecal indicator bacteria in surf zone off Huntington Beach, CA. Marine Environm. Res. 61:471-493.

25. SOLOMON, C.T.; CARPENTER, S.R.; CLAYTON, M.K.; COLE, J.J; COLOSO, J.J; PACE, M.L.; ZANDEN, M.J.; WEIDEL, B.C. 2011. Terrestrial, benthic, and pelagic resource use in lakes: results from a three-isotope Bayesian mixing model. Ecology. 92(5):1115-1125.

26. SHIBATA, T.; SOLO-GABRIELE, H.; FLEMING, L.; ELMIR, S. 2004. Monitoring marine recreational water quality using multiple microbial indicators in an urban tropical environment. Water Res. 23:3119-3131.

27. SULLIVAN, T.; SNYDER, K.; GILBERT, E.; BISCHOFF, J.M.; WUSTENBERG, M.; MOORE, J.; MOORE, D. 2005. Assessment of Water Quality in Association with Land Use in the Tillamook Bay Watershed, Oregon, USA. Water, Air Soil Pollut. 161:3-23.

28. VELÁSQUEZ R, J.O; JIMÉNEZ G., G.J.; SEPULVEDA L., M. 2007. Determinación de la calidad ambiental de la Ciénaga de Colombia. Gestión y Ambiente. 10(1):187200.

Recibido: Agosto 27 de 2010

Aceptado: Noviembre 1 de 2011 